\title{
Measurement of the Validity and Reliability of the Persian Translation of the Saint George Respiratory Questionnaire for Patients with Chronic Obstructive Pulmonary Disease
}

\author{
Saeid Fallah Tafti ${ }^{*}, 1,3,4$, Ali Cheraghvandi ${ }^{1,3,4}$, Mehran Marashian ${ }^{1,3,4}$, \\ Habib Emami ${ }^{2,3,4}$ and Bahareh Mokri ${ }^{1,3,4}$
}

\author{
${ }^{I}$ Department of Internal Medicine, and ${ }^{2}$ Department of Epidemiology, National Research Institute of Tuberculosis and \\ Lung Disease (NRITLD), Iran \\ ${ }^{3}$ Masih Daneshvari Hospital, Iran \\ ${ }^{4}$ Shahid Beheshti University of Medical Sciences, Iran
}

\begin{abstract}
Background: One of the important tools in measuring the quality of life in patients having a chronic disease is using questionnaires in which the impact of disease and its different types of treatments on daily tasks and social activities are evaluated.

Materials and Methods: At first, the questionnaire was translated into Persian and some of the concepts were changed by the researchers. Next, it was back translated by a person living in an English-speaking area who was unaware of the subject. At the fourth stage, the concepts were revised by a group of 15 patients and then 55 patients filled out the questionnaire. Finally, it's internal consistency was evaluated by calculating the Cronbach's alpha coefficient.

Results: After translation of the questionnaire, the researchers modified $17 \%$ of the sentences and phrases according to the Iranian culture. Questions which were controversial and those for which appropriate proposals were made by the COPD patients were ultimately modified and were responded to by an additional group of 55 patients. Statistical analysis and internal consistency of the questions in the different sections were obtained. For the first section ("complaints") and following the deletion of the last question (which was in regards to wheezing), the Cronbach's alpha was equal to 0.74. In the second section, where the patients' physical activities and the impact of disease on them were evaluated, the Cronbach's alpha coefficient was equal to 0.93 . The last question of this section concerning the impact of activity on the rate of respiratory difficulty was deleted due to heterogeneous choices and unresponsiveness of 5 patients (15\%). As a result, the Cronbach's alpha coefficient rose to 0.95 on this section and the Cronbach's alpha coefficient for the entire questionnaire was calculated at 0.93 .

Conclusion: In addition to conformity with the concepts of the English version, the translated Persian version of this questionnaire approximately matches the Iranian culture and can be used for the evaluation of the quality of life in chronic respiratory patients.
\end{abstract}

Keywords: Questionnaires, respiratory diseases, reliability and validity, quality measurement.

\section{INTRODUCTION}

During the past 20 years, there have been several studies in regards to the evaluation of quality of life on the basis of different questionnaires which has considerably improved health care services. Pulmonary disorders, particularly COPD are some of the most debilitating diseases of people. Considering the vast impact of these disorders on the quality of life as well as the mood and attitude of patients, treatment is valuable only if the effect of the disorder on them and their daily activities can be evaluated. Today, several methods exist to evaluate health and quality of life and one of the best

*Address correspondence to this author at the Department of Internal Medicine, National Research Institute of Tuberculosis \& Lung Disease (NRITLD), Masih Daneshvari Hospital, Darabad, Shaheed Bahonar (Niavaran) Ave. Post Code: 1955841452, P.O. Box: 19575/154, Tehran, Iran; Tel: +98-21-20109502; Fax: +98-21-20109502;

E-mail: saeid_ftafti@yahoo.com and cheapest of methods is using self completing questionnaires.

COPD is a worldwide disease so assessment of quality of life in such patients is very important. But, same questionnaires are understandable with different meanings by variable cultures. The St. George questionnaire as a tool for quality of life assessment has been translated in several languages. Persian translation of St. George questionnaire based on Iranian culture is the novelty of this study.

Usually, there are two ways to evaluate health: general questionnaires in which the general health status and quality of life are evaluated and disease-specific questionnaires that are designed to evaluate a specific aspect of life [1]. The quality of life has already been extensively evaluated with a variety of goals in mind such as determination of the intensity and prognosis of disease, evaluation of health and effectiveness of rehabilitation [2-8] and the St. George 
questionnaire is a widely known and simple and standard tool for evaluating different aspects of health in COPD patients [9].

This questionnaire has been translated into 35 different languages and even edited according to English-American concepts [10]. Considering the validity and reliability of the concepts, the Spanish version of this questionnaire was not significantly different from the English version $[9,11]$. This questionnaire consists of 50 questions and 76 items of evaluation and has been designed in three sections. The first section includes sequence and intensity of respiratory complaints. The second includes activities that are restricted and can lead to dyspnea and the third section includes impact of a chronic respiratory disorder on social activities and psycho-social issues. Each section is scored from 0 to 100 where 0 shows quite healthy. This questionnaire is quite easy to fill-in within 10 minutes and it's validity has been accepted repeatedly in COPD and asthmatic patients [12, 14].

The most important factors necessitating for preparation of the Persian version of the St. George questionnaire are as follows:

A) Improvement of the quality of the Persian version of the St. George questionnaire in order to evaluate the quality of life in COPD patients and to modify it according to the Iranian culture in a way that changing the structure of the sentences is avoided and it perfectly matches the original version.

B) Determination of validity and reliability of this trend in order to prevent misleading results. The most important point in an applied valid questionnaire is the degree of its validity and reliability. These two factors depend heavily on the rate of response by the patients and repeatability of the responses. The questions must be written in a way that patients can understand and compare the situation with their own. This will contribute to an increase in the accuracy of the responses and therefore, the results will statistically support the content validity and internal consistency which in turn show the questionnaire's validity and reliability. Frequent surveys and evaluation of the responses and comparing them with the quality of health and situation of the patient is very helpful in preventing deviation from the main purpose $[9,11,12]$.

C) Thus, this study was conducted in COPD patients referred to Dr. Masih Daneshvari Hospital, Tehran, Iran with the aim of determining the validity and reliability of the Persian version of the St. George questionnaire.

\section{MATERIALS AND METHODS}

The original version of the St. George questionnaire in English was used in this study. COPD severity was considered according to GOLD criteria [15]. Patients with an atopy history, episode of cough and dyspnea relief with treatment or spontaneously and also improvement in forced expiratory volume in first second (FEV1) $>20 \%$ after $\beta 2$ agonist inhalation, seasonal allergies, allergic rhinitis and eczema were supposed to be suffered from asthma and were excluded from this project whereas COPD is characterized by age over 40 years with a history of cough, sputum and dyspnea episodes for at least 2 years and also history of smoking. So, history of the patients in addition to spirometry were the key diagnostic tools to distinguish the COPD from asthmatic patients $[16,17]$. No confidential or personal questions are asked in this questionnaire. Therefore, the patients' rights have been completely observed. The participants received information about the study. Consent was obtained of participants. The questionnaires were completed in the ward by the patients with sufficient literacy or by the asker if the patients haven't enough literacy. The family members were not allowed to assist the patients. Most of the patients had middle level of literacy and socioeconomic state. The questionnaire included 52 questions regards to 3 main subjects including symptoms, activities and impact. It took 10 minutes to fill in the questionnaire. The content of the questionnaire was separately evaluated and finally confirmed by a pulmonologist, 2 general internists, an epidemiologist and a general practitioner.

In order to translate the questionnaire into Persian, we used the standard Brislin's method as follows:

1) Forward Translation: A translator expert in both Persian and English languages who has a degree and experience in the medical sciences translated the questionnaire into Persian. Accuracy and fluency of the translated text was emphasized in order to enable the patients to read and comprehend the questions easily.

2) Review: In this stage, the Persian version of the questionnaire was reviewed by some Iranians to correct the mistakes in case there were any.

3) Backward Translation: In this stage, the completed and corrected Persian version of the questionnaire was again translated into English. The translator was not at all familiar with the questionnaire and merely translated the sentences into English. This unfamiliarity helped in showing the flaws and faults of the Persian version.

4) Comparison: In this stage as final stage, the backward translated questionnaire was compared with the original version and the differences were surveyed and then modified. The differences between these two versions showed that the Persian translation had not been done skillfully and thus, it was modified in a way that the backward translated version was at the maximum level of similarity to the original version.

\section{Scoring}

Scoring of the St. George questionnaire is from $0 \%$ to $100 \%$ where $0 \%$ is considered as the best health situation of all choices and $100 \%$ is considered as the worst. The intermediate choices of any question were scored from 0 to 100 according to the number of choices and proportionately. For instance, in a question with 5 multiple choices the scores were $0,25,50,75$ and 100 . The scores of all the questions in each section were summed and the total score for the questionnaire was obtained by adding the section's scores. 


\section{The Pilot and Final Studies}

Conducting a pilot study on some of the patients was necessary in order to consider their opinion directly about the Persian questionnaire and reach a desirable result.

A pilot study was conducted on 15 COPD patients hospitalized at the Masih Daneshvari hospital in May 2005. The results showed that some of the questions were not understandable by the patients and some others were culturally disproportionate to the Iranian patients. For example, few people walk with their dogs or play golf in Iran. Therefore, some of the questions were modified according to the Iranian culture and some others were omitted.

The main study was conducted on 55 COPD hospital patients at the Masih Daneshvari hospital following preparation of the final Persian version of the St. George questionnaire. The study was cross-sectional and was performed in September 2005. Sampling was easily available and hospitalized patients were respectively included in the study. The Persian version of the St. George questionnaire and also the Katz questionnaire (indices for evaluating patients' physical abilities) were administered and fully explained to the patients so that they were able to answer the questions on their own.

\section{Statistics and Data}

Data was collected through scoring the St. George questionnaire. This information with demographics and the scores obtained from the Katz questionnaire were analyzed by SPSS-13 software. The Cronbach's alpha coefficient was obtained to compare the findings and to determine the internal consistency of the questionnaire.

\section{RESULTS}

A total of 55 patients, 3 women and 52 men, were included in the study. The mean age was $66.4 \pm 11.1$ years with a minimum of 40 and maximum of $88.13(24 \%)$ of the included patients had moderate and $42(76 \%)$ had severe COPD based on GOLD criteria. No of the included patients had mild COPD. The Cronbach's alpha coefficient was needed for any section in order to determine the internal consistency of the Persian version of the St. George questionnaire. This coefficient was 0.74 for "symptoms" and although it was above $70 \%$ and therefore acceptable for internal consistency, question number 8 (the last question of the "symptoms" section) was omitted as few patients had correctly answered it. Following this change, the Cronbach's alpha rose to 0.78. It should be noted that a Cronbach's alpha coefficient above 0.70 indicates a desirable efficiency and internal consistency in such studies.

The Cronbach's alpha coefficient was 0.93 for the second and third sections ("physical activities" and "impact") which is excellent. In this section, since the last question in regards to the impact of physical activity on dyspnea was responded to by 5 patients $(15 \%)$, it was deleted and the Cronbach's alpha rose to 0.95 . Finally, the Cronbach's for the entire questionnaire, after deleting the inappropriate questions, reached 0.93 .

Comparison of the score for the "activities" section of the questionnaire with the Katz score which is a standardized questionnaire for evaluation of physical abilities showed a correlation of $-0.50(p<=0.01)$ and is significant. The symbol " -“ (minus) shows opposite scoring in the two questionnaires; i.e. a patient having the least activity and the worst general condition would get the highest score on St. George and the lowest on Katz. It should be noted that the Katz questionnaire is related to quality of life and in order to find a correlation between the scores of the two questionnaires, the "symptoms" section had to be ignored as consideration of this section in the assessment of quality of life would lead to statistical error.

\section{DISCUSSION}

The Saint George Respiratory Questionnaire is specific for patients with obstructive pulmonary disease and is filled out by the patients themselves. This questionnaire was developed by Jones and his colleagues and has 50 questions in three sections of "symptoms," "activity," and "Impact."

Many researchers have already started to translate the St. George questionnaire into different languages and study its validity. In a study conducted by Ferrer et al. on 318 male COPD patients in 1996, this questionnaire was translated into Spanish and then used. Concurrently, several other health tests were performed and the results of the tests were compared with the findings of the St. George questionnaire. Ferrer et. al. concluded that the Spanish version of the St. George questionnaire was acceptable and easy to answer. The Cronbach's alpha coefficient was 0.94 which shows a very nice internal consistency [11]. The results of this study show that the scores of the "activity" and "impact" sections of the St. George questionnaire are comparable with the Nottingham Health Profile (NHP) scores, but the score on the "symptoms" section is not since the NHP is designed according to the patients' activities and performance not their complaints [12-18]. One of the main problems of the study conducted by Ferrer et al. was that some of the questions were not understandable by the patients and therefore, there was no reliable answers for them. Another problem was in regard to questions that had to be left unanswered in case there were no specific symptoms; yet, they were actually answered by the patients. In addition, several problems arose since only COPD patients were included in the study and some of the questions were specific to asthmatics.

Curtis et al. cited that the St. George questionnaire has shown a high level of validity and reliability in their study [19].

Another study which was performed on the American version of the St. George questionnaire showed acceptable internal consistency with a Cronbach's alpha coefficient higher than 0.70 (except for the "complaints" section]. Reliability of this questionnaire, following the test-retest comparison, showed a number between 0.795 and 0.9 and validity of this questionnaire in comparison with other pulmonary functional indices such as classification of dyspnea according to MRC or SF-36 was quite acceptable $(\mathrm{p}<=0.01)[10]$.

Jones et al. noticed that demographic factors and other disease related factors had the least impact on weighing of the questions. Furthermore, Quirk's study showed that in different countries, the findings related to asthmatic patients 
had made a relatively low difference in weighing of the questions. Thus, it can be concluded that the St. George questionnaire can efficiently be used in an extensive range of chronic pulmonary diseases from asthma to COPD. Jones et al. concluded that the St. George questionnaire is a valid tool in quantitatively measuring symptoms of patients and their quality of life.

In 2002, following the pilot study and translation of the questionnaire into Spanish in 1996, Ferrer and Villasante and colleagues studied the quality of life in healthy people. During the study, the researchers found out that even people in the best conditions do not achieve the lowest score (zero), because it is likely that they have some symptoms or limitations of activity and/or they may interpret their own quality of life in a wrong way. They also noticed that in contrast to Jones' study, people of different genders and ages who obtained the same score did not have the same quality of life. For example, a 45 year-old person with a score of 60 is in a worse condition than a person with the same score who is 65 years old. This is because in a healthy community, the elderly and also the female individuals are relatively more restricted due to reasons other than COPD. Clearly, the elderly can not be as active as younger individuals. As younger individuals likewise, due to cultural issues, women have more restrictions than men do. In a study conducted by Ferrer, they had less pulmonary symptoms and yet, their activity scores were higher showing worse situations. After comparing them to a healthy population, these differences were perfectly understood. People belonging to different socioeconomic classes had different scores which were eliminated following correction for this factor. Nonetheless, differences related to the level of education could not be eliminated. Ferrer suggests that using the criteria from a healthy society for comparison is very helpful in evaluating quality of life and will allow researchers to benefit from more documented, more acceptable and more applicable results.

Wilson et al. conducted a study on 111 bronchiectatic patients under evaluation with the St. George questionnaire. These researchers pointed out that since they had no tools to evaluate quality of life in patients having bronchiectasis, they used the St. George questionnaire which is designed for asthmatic and COPD patients. Besides filling the St. George questionnaire, their patients had been evaluated in regards to general health, mood and body weakness. Findings related to the St. George questionnaire and clinical and mental findings of the patients at the time of reference were compatible with those of follow-up. Cronbach's alpha coefficient for the St. George questionnaire was 0.97 which shows a good internal consistency. In this study, repeatability was evaluated in 23 patients in a 2 week interval and the results were acceptable. Wilson showed that the St. George questionnaire has a good convergence with some clinical evaluation tools in terms of the activity of patients and severity of the disease in a patient with bronchiectasis.

In the present study, despite the controversial results observed by Ferrer and Jones regarding the effects of demographic factors and respiratory-related factors in filling and scoring on the St. George questionnaire, the author attempted to ignore these controversies. Thus, patients were not separated according to their gender, age, culture and other demographic factors and the final score was the only criterion for evaluating the quality of life since the objective of the current study is to translate and determine the validity and reliability of the Persian version of the St. George questionnaire. In our study, the high Cronbach's alpha coefficient well shows the internal consistency. Nevertheless, our patients had some restrictions in filling the questionnaire. Many of the questions in the field of activity were not compatible to the Iranian culture and had to be modified. For instance, walking the dog or going to church were replaced by walking in the park or going to the mosque. Besides, one of the questions regarding job problems was to some extent problematic in our pilot study since our patients were mostly retired men or house wives and therefore, it was deleted. One of the questions in the English version was about the negative impact of the disease on recreational activities or hobbies which was also deleted in the statistic analysis because it seemed unfamiliar and meaningless to the patients and the responses were not reliable enough. Therefore, the Cronbach's alpha coefficient and internal consistency were finally increased to 0.93 . Ultimately, the Persian version of the St. George questionnaire was designed in three sections of "symptoms," "activities" and "impact" on performance with 50 questions including 7 for "symptoms" and 43 for "activities" and "impact" on performance. This can be used in more extensive studies with more detailed evaluations and can be compared to pulmonary function tests, physical activity tests and mental health measurement tools like as was done by Wilson.

In the present study, According to more smoking in men than women in our country, most of our included patients were males. Thus, based on this study, validity and reliability of Persian version of St. George questionnaire can be extended to male group. More researches are needed to study if the Persian St. George questionnaire can be useful in female group.

\section{ACKNOWLEDGEMENT}

The authors would like to thank Dr. Firouzeh Talischi for edition of this manuscript.

\section{REFERENCES}

[1] Lohr KN. Advances in health status assessment. Overview of the conference. Med Care 1989; 27(Suppl 3): S1-11.

[2] Meek PM, Lareau SC. Critical outcomes in pulmonary rehabilitation: assessment and evaluation of dyspnea and fatigue. $\mathrm{J}$ Rehabil Res Dev 2003; 40(5 Suppl 2): 13-24.

[3] Jones PW, Quirk FH, Baveystock CM, Littlejohns P. A selfcomplete measure of health status for chronic airflow limitation. Am Rev Respir Dis 1992; 145(6): 1321-7.

[4] Wedzicha JA, Bestall JC, Garrod R, Garnham R, Paul EA, Jones PW. Randomized controlled trial of pulmonary rehabilitation in severe chronic obstructive pulmonary disease patients, stratified with the MRC dyspnoea scale. Eur Respir J 1998; 12(2): 363-9.

[5] Bestall JC, Paul EA, Garrod R, Garnham R, Jones PW, Wedzicha JA. Longitudinal trends in exercise capacity and health status after pulmonary rehabilitation in patients with COPD. Respir Med. 2003; 97(2): 173-80

[6] Griffiths TL, Burr ML, Campbell IA, et al. Results at 1 year of outpatient multidisciplinary pulmonary rehabilitation: a randomised controlled trial. Lancet 2000; 355(9201): 362-8.

[7] Finnerty JP, Keeping I, Bullough I, Jones J. The effectiveness of outpatient pulmonary rehabilitation in chronic lung disease: a randomized controlled trial. Chest 2001; 119(6): 1705-10.

[8] Incalzi RA, Bellia V, Catalano F, et al. Evaluation of health outcomes in elderly patients with asthma and COPD using disease- 
specific and generic instruments: the salute respiratoria nell'anziano (Sa. RA) study. Chest 2001; 120(3): 734-42.

[9] Wilson CB, Jones PW, O'Leary CJ, Cole PJ, Wilson R. Validation of the St. George's respiratory questionnaire in bronchiectasis. Am J Respir Crit Care Med 1997; 156(2): 536-41.

[10] Barr JT, Schumacher GE, Freeman S, LeMoine M, Bakst AW, Jones PW. American translation, modification, and validation of the St. George's Respiratory Questionnaire. Clin Ther 2000; 22(9): 1121-45.

[11] Ferrer M, Alonso J, Prieto L, et al. Validity and reliability of the StGeorge's respiratory questionnaire after adaptation to a different language and culture: the Spanish example. Eur Respir J 1996; 9(6): 1160-6.

[12] Jones PW. Health status measurement in chronic obstructive pulmonary disease. Thorax 2001; 56(11): 880-7.

[13] Jones PW. Quality of life, symptoms and pulmonary function in asthma: long-term treatment with nedocromil sodium examined in a controlled multicentre trial. Nedocromil sodium quality of life study group. Eur Respir J 1994; 7(1): 55-62.
[14] Jones PW, Bosh TK. Quality of life changes in COPD patients treated with salmeterol. Am J Respir Crit Care Med 1997; 155(4): 1283-9.

[15] Petsonk EL, Hnizdo E, Attfield M. Definition of COPD GOLD stage I. Br Med J 2007; 62(12): 1107-8.

[16] Kohler D, Fischer J, Raschke F, Schonhofer B. Usefulness of GOLD classification of COPD severity. Thorax 2003; 58(9): 825.

[17] Martinez FJ, Standiford C, Gay SE. Is it asthma or COPD? The answer determines proper therapy for chronic airflow obstruction. Postgrad Med 2005; 117(3): 19-26.

[18] Alonso J, Anto JM, Gonzalez M, Fiz JA, Izquierdo J, Morera J. Measurement of general health status of non-oxygen-dependent chronic obstructive pulmonary disease patients. Med Care 1992; 30(Suppl 5): 35 .

[19] Quirk FH, Jones PW. Patientsúperception of distress due to symptoms and effects of asthma on daily living and an investigation of possible influential factors. Clin Sci 1990; 79(1): $17-21$

(C) Tafti et al.; Licensee Bentham Open.

This is an open access article licensed under the terms of the Creative Commons Attribution Non-Commercial License (http://creativecommons.org/licenses/by$\mathrm{nc} / 3.0 /$ ) which permits unrestricted, non-commercial use, distribution and reproduction in any medium, provided the work is properly cited. 